\title{
Respiratory distress due to platypnea-orthodeoxia syndrome: A diagnostic challenge in emergency setting
}

\author{
Sandro Di Domenico, ${ }^{1}$ Paolo Aseni, ${ }^{1}$ Luca Bonacchini, ${ }^{1}$ Maurizio Bottiroli, ${ }^{2}$ Rossella Guerrieri, ${ }^{1}$ \\ Adriano Basile, ${ }^{1}$ Andrea Bellone ${ }^{1}$ \\ ${ }^{1}$ Emergency Department and Urgency Medicine Ward; 'Anesthesia and Critical Care "De Gasperi" Cardio \\ Center, Niguarda Hospital, Milan, Italy
}

\begin{abstract}
Platypnea-orthodeoxia syndrome (POS) is an unusual cause of hypoxia characterized by enormous variation in oxygen saturation during postural changes. We describe here the clinical presentation, the diagnostic challenge of POS and discuss the main pathophysiological etiologies of the disease in a 75-year-old woman who was admitted because shortness of breath. After ruling out the most important causes of dyspnea we observed that the symptoms improved when the patient was lying flat. The diagnostic workup with computed tomography scan and air bubble saline echocardiography lead us to a correct diagnosis.
\end{abstract}

\section{Introduction}

Platypnea-orthodeoxia syndrome (POS) is an uncommon and underdiagnosed cause of hypoxia characterized by huge variation in oxygen saturation during postural changes. It was first described by Burchell ${ }^{1}$ in the late $1940 \mathrm{~s}$ in a patient with post-traumatic arteriovenous shunt and afterward named platypnea (positional dyspnea) and orthodeoxya (arterial desaturation in standing position). Because of the rarity of POS, only a few case series reporting

Correspondence: Luca Bonacchini, Emergency Department and Urgency Medicine Ward, Niguarda Hospital, Piazza Ospedale Maggiore 3, 20192 Milan, Italy.

Tel.: +39.0264447433.

E-mail: luca.bonacchini@ospedaleniguarda.it

Key words: Platypnea; Respiratory distress; Patent foramen ovale; Orthodeoxia; Ascending aortic aneurysm.

Contributions: the authors contributed equally.

Conflict of interest: the authors declare no potential conflict of interest.

Funding: none.

Received for publication: 28 December 2017.

Revision received: 21 February 2018.

Accepted for publication: 19 March 2018.

This work is licensed under a Creative Commons Attribution 4.0 License (by-nc 4.0).

CCopyright D. Di Sandro, 2018

Licensee PAGEPress, Italy

Emergency Care Journal 2018; 14:7265

doi:10.4081/ecj.2018.7265 patient outcomes have been published. POS is defined by the occurrence of dyspnea and hypoxia (a drop in oxygen saturation bigger than $5 \%$ or a drop in $\mathrm{PaO}_{2}>4 \mathrm{mmHg}$ when changing position from supine to upright position) and by the observation of symptoms resolution with recumbence. It is the consequence of different physio-pathological pathways causing an increase of arteriovenous shunt while the patient is standing. Here we report our experience of managing a patient with POS and right-to-left atrial shunt associated with patent foramen ovale (PFO).

\section{Case Report}

A 75-year-old woman was admitted to our Emergency Department, because of shortness of breath without chest pain or cough. She had a history of glaucoma, hernioplasty, hysterectomy and endoscopic excision of anal polyposis. She had been ill for the last three months with several episodes of progressive dyspnea. She was admitted twice to a local hospital, and the following tests were obtained: a contrast computed tomography (CT) scan of the chest revealing no signs of pulmonary embolism, lung consolidation or pleural effusion; a coronary angiography with no evidences of critical coronary stenosis; pulmonary functional tests within the normal reference values; trans-thoracic echocardiography showing a mild mitral insufficiency, moderate-severe aortic valve insufficiency, and moderate enlargement of the ascending aorta (Figure 1). She was discharged with oral steroid, loop diuretics, and proton pump inhibitor.

On physical examination she was tachypneic and in severe respiratory distress, peripheral oxygen saturation $\left(\mathrm{SpO}_{2}\right): 77 \%$ on room air, blood pressure $115 / 70 \mathrm{mmHg}$. The heart rate was regular, 108 beats per minute, a 3/6 systo-diastolic murmur was audible. Auscultation of the chest revealed a slight reduction of respiratory sound without any pathological findings.

An arterial blood gas analysis while breathing room air showed severe hypoxemic, hypocapnic respiratory alkalosis ( $\mathrm{pH}: 7.54$, $\mathrm{pCO}_{2}: 28 \mathrm{mmHg} ; \mathrm{pO}_{2}: 32 \mathrm{mmHg} ; \mathrm{HCO}_{3}: 24 \mathrm{mmol} / \mathrm{l} ; \mathrm{SpO}_{2}: 71 \%$ ).

She was admitted to our Emergency Medicine ward, and we were puzzled by the huge variations during the same day of her oxygen saturation, that was ranging from $79 \%$ to $97 \%$ on ambient air. Despite some episodes of severe dyspnea crisis, she seemed to tolerate well low blood oxygen levels. She was treated with i.v. steroid, bronchodilators, antibiotics and Non- Invasive Mechanical Ventilation obtaining no remarkable improvement. We noticed that the symptoms improved when the patient was lying flat (thus excluding the clinical diagnoses of heart failure).

Due to the increasing episodes of respiratory distress, unresponsive to medical therapy and oxygen supplementation she was transferred to the Intensive Cardiology Unit where a severe drop in the oxygen saturation was confirmed on postural changing from 
$95 \%$ when lying to $79 \%$ in the upright position.

The finding of hypocapnic hypoxia was consistent with an arteriovenous shunt (AVS) as the primary physio-pathological drive, so we tried to rule out the main causes of AVS. A contrastenhanced CT scan of the chest was performed. It showed no signs of pulmonary embolism, no mass, no pleural fluid and no pneumonia; the diameter of pulmonary artery was $32 \mathrm{~mm}$, close to the upper limit of the normal range; the ascending aorta was dilated (maximum diameter $48 \mathrm{~mm}$ ). A right heart catheterization revealed Pulmonary artery (PA) systolic pressure $17 \mathrm{mmHg} / \mathrm{PA}$ diastolic pressure $7 \mathrm{mmHg}$ / average PA $11 \mathrm{mmHg}$, Wedge Pressure 11 $\mathrm{mmHg}$, with a right normal oximetry; no left heart oxygen samples were taken. The six-minute walking test revealed low tolerance to exertion and rapid desaturation, but no remarkable difference was registered when the patient was receiving or not oxygen supplementation. Repeated pulmonary function tests were normal. A transthoracic echocardiography in the sitting position using microbubble contrast revealed the presence of significant early opacification of left atrium consistent with a severe intracardiac shunt. A transesophageal echocardiogram confirmed the diagnosis showing a huge PFO with a right to left shunt and demonstrated a compression and distortion of the right atrium.

Reassessing the images of the CT scan of the chest (Figure 2) we noticed the whole architecture of the heart was changed with the right atrium compressed by the dilated aortic root possibly causing a redirection of the blood flow from inferior vena cava through the atrial septum. The standing position might have increased the area of the PFO by stretching the atrial septum. We then speculated that the right-to-left interatrial shunt could be secondary to the enlarged PFO area and the perfect alignment between the vena cava blood flow and the left atrium overcoming the higher pressure of left atrium in the absence of significant pulmonary hypertension.

In consideration of the worsening of the clinical picture, the patient underwent a combined cardio-surgical procedure consisting of PFO closure, aortic valve and aortic root replacement. Postoperative course was complicated by atrioventricular block, and a Pacemaker was implanted and the patient was discharged after 12 days without any episode of respiratory distress. At the one-year follow-up after surgery, the patient was in good clinical conditions.

\section{Discussion}

The literature about POS is mainly based on case reports and small series; Agrawal et al. ${ }^{2}$ recently reviewed literature finding that $83 \%$ of the cases were secondary to an intra-cardiac shunt. The etiology of POS can be divided into four major groups: intra-cardiac shunt, pulmonary shunt, ventilation-perfusion mismatch or a combination of these as shown in Table 1.

Intra-cardiac shunt is generally consequent to PFO; less common causes are atrial septal defect and atrial septal aneurysm; the latter being more often associated with anatomical or functional alterations. In contrast to the usual findings, POS can be associated with a right to left intra-cardiac shunting in the absence of any demonstrable pressure gradient between right and left atria. ${ }^{3,4}$ Right to left shunting despite normal right atrial pressure has also been reported after right pneumonectomy $y^{5,6}$ or in association with venous embryonic remnants. In these situations, an altered anatomical relation between the superior and inferior vena cava and the atrial septum presumably accounts for flow-related rather than pressure-related shunting. ${ }^{7,8}$
Some rare causes of POS such as pulmonary arteriovenous malformations and hepato-pulmonary syndrome should also be considered. Arteriovenous malformations usually occur at the bases of the lung. The entity of the shunt increases when patient is standing because the perfusion of lower areas of the lungs increases with gravity. Other causes of POS are related to ventilation-perfusion mismatch. Upper parts of the lungs are well ventilated but badly perfused, on the contrary, lower parts are well perfused. A consolidation or a mass affecting the bases can alter the ventilation-perfusion ratio, leading to a reduction of oxygen saturation. When the patient is lying the redistribution of the areas with good

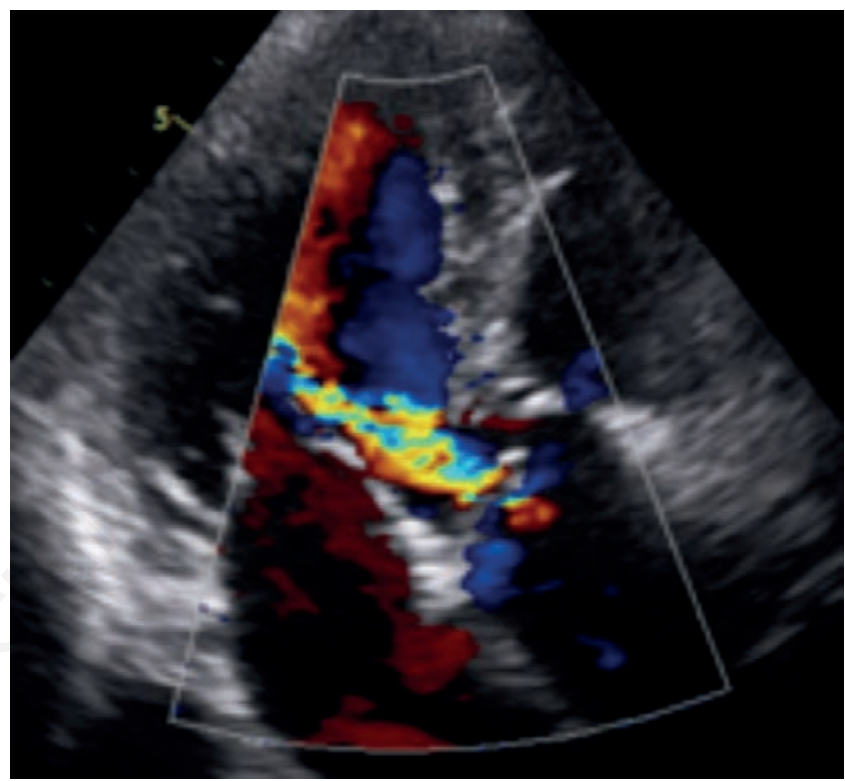

Figure 1. Moderate-severe aortic regurgitation jet from Parasternal Long Axis view.

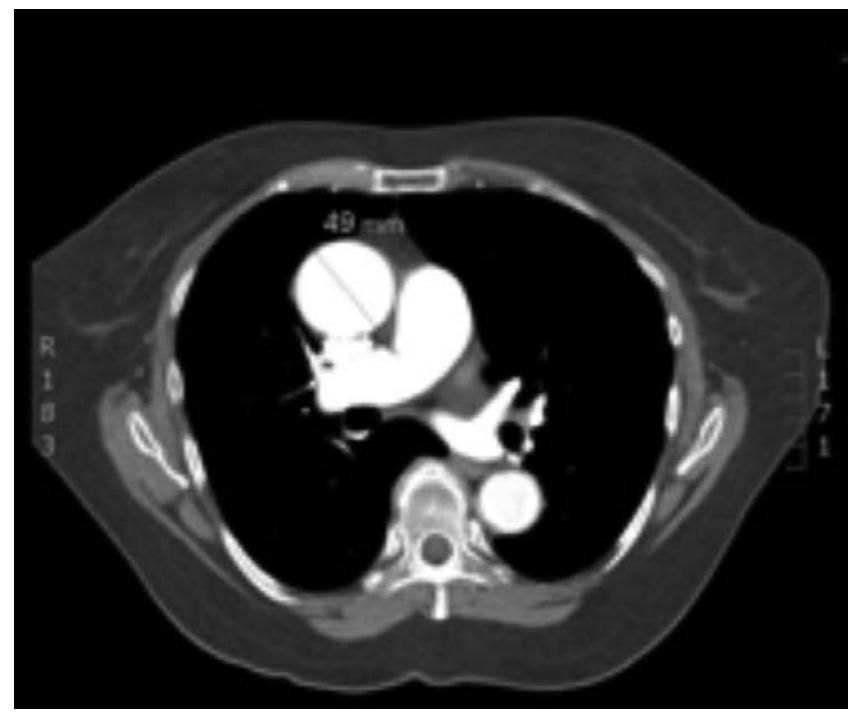

Figure 2. Chest computed tomography scan showing no signs of pulmonary embolism and ascending aortic aneurysm. 
ventilation-perfusion ratio can lead to a more favorable saturation. The hepato-pulmonary syndrome is a condition that involves both the liver and lungs. The syndrome is characterized by a triad of symptoms: hypoxemia in standing position, intrapulmonary vascular dilatations, and a chronic liver disease. Embolization of large porto-pulmonary channels when evident and orthotropic liver transplantation could be the only treatment of choice for the most severe cases, whereas sildenafil can be useful in porto-pulmonary hypertension. ${ }^{9}$

Table 1. Clinical conditions associated with platypnea-ortodoxya syndrome.

\section{CARDIAC DISEASES}

Atrial septal defect

Atrial septal aneurysm

Aortic aneurysm

Constrictive pericarditis

Eosinophilic endomyocardial disease

Patent foramen ovale

Persistent Eustachian valve or Chiari network

Pericardial effusion

Right atrial mass

PULMONARY DISEASES

Diaphragmatic paralysis

Chronic obstructive pulmonary disease

Pulmonary fibrosis

Pulmonary emphysema

Pneumectomy

Lobectomy

Hepatopulmonary syndrome

Blunt chest wall trauma

Idiopathic hemidiaphragmatic paralysis

Single lung transplantation

Severe kyphosis

Paraesophageal hernia

Hemidiaphragmatic paralysis

Blunt chest trauma

ABDOMINAL DISEASES

Hepatic cirrhosis with porto-pulmonary shunt

Ileus

Ileus post- abdominal surgery

Large hepatic Hydatid cyst

Adult policystic liver diasease

MISCELLANEOUS DISEASE

Kyphoscoliosis

Thoracic vertebral fracture

Autonomic neuropathy

Parkinson's disease

Organophosphorus poisoning

Radiation-induced bronchial stenosis.

Traumatic bronchial rupture

Ileus

Fat embolism
Different intrapulmonary and extra-pulmonary causes of arteriovenous shunt were considered in our patients. With i.v. contrast CT scan, we easily rule out the commonest causes of intrapulmonary shunt like pulmonary embolism, arteriovenous malformations and other parenchymal or anatomical alterations leading to ventilation/perfusion mismatch. A standard transthoracic echocardiogram failed to reveal an intra-cardiac shunt and showed no signs of pulmonary hypertension; trans-thoracic echocardiogram is not the gold standard for the diagnosis of intra-cardiac shunt, but sometimes it can reveal its presence showing Doppler signal across the interatrial wall. If we consider that echocardiography is usually performed with the patient lying on the bed, this information can be even harder to obtain as in our patient where symptoms were evident only in the upright position. The best diagnostic test to demonstrate right to left shunt is the echocardiography with microbubble contrast. The early presence of microbubbles in the left atrium is suggestive for intra-cardiac shunt, while a delayed appearance of the contrast is consistent with extra-cardiac shunt that in most of the cases is intrapulmonary. ${ }^{10}$

Some cases of aneurysm of the ascending aorta associated with right to left shunting across a PFO have been reported during last years. ${ }^{11-14}$ In a series of 18 patients with POS and PFO, ${ }^{15} 4$ patients had an associated aortic root aneurysm, and 12 patients presented an enlarged aortic root. Since the right atrial pressure is lower than the left one even in the presence of PFO, no right to left shunt is generally possible. However, the association of PFO with aortic root enlargement and the consequent anatomical remodeling of the mediastinal structure, can lead to a redirection of the inferior cava vein flow through the PFO. Several other mechanisms have been hypothesized. An aneurysm of the aortic root may increase the stretch effect over the septum in standing position causing a widening of PFO area. Eicher et al. ${ }^{15}$ speculated that an enlarged or horizontalized aortic root might interfere with the atrial septum. This can cause a rightward shift of the fossa ovalis and a narrowing of the angle between atrial septum and inferior vena cava with the blood flow and the atrial defect into a closer relation. Furthermore, the bulging of the aortic root above the tricuspid orifice, sometimes completed by the presence of a Eustachian valve, may behave functionally like an acquired cor triatriatum dexter. In this respect, some evidence on ultrasound series demonstrates that an enlarged aortic root reduces the distance between the aorta and the posterior atrial wall, thus decreasing the tautness of the atrial septum. ${ }^{15}$ The latter mechanism could allow the flap valve of the fossa ovalis to move more freely and in the presence of a normal left-right atrial pressure gradient it may lead to a spinnaker effect which maintains the PFO wide open. Finally, the compression of the aortic root may reduce the right atrial compliance due to its smaller size.

In our patient, contrast-enhanced CT scan and the transthoracic US revealed the modification of the usual anatomical relations of the mediastinal vessels with cardiac chambers. In accord with other reports, we hypothesized that the aortic enlargement might have squeezed the right atrium causing the alignment of inferior vena cava vein flow with PFO. For this reason, closure of PFO and removing the secondarily associated alterations was considered the only possible surgical option.

\section{Conclusions}

The diagnosis of POS is based on high level of suspicion when the patient presents a huge variation of oxygen saturation from lying to the upright position. Arterial blood gas analysis reveals 
hypoxia and hypocapnia consistent with right to left shunting. Although cardiac catheterization can demonstrate a mismatch in oxygen saturation between pulmonary vein and aorta and can be helpful in excluding pulmonary hypertension, this diagnostic procedure is usually performed with patient lying, when shunt is inactive and this can represent a confounding bias of the procedure. Bubble contrast Echocardiography is the gold standard test that can easily reveal the right to left shunt. It is important to underline that the test should be performed with patient either lying and in sitting position. The appearance of the bubbles in the left heart within 3 cardiac cycles suggests an intracardiac shunt, while a delayed contrast is more consistent with an extra cardiac shunt.

\section{References}

1. Burchell HB, Helmholz HF Jr, Wood EH. Reflex orthostatic dyspnea associated with pulmonary hypotension. Am J Physiol 1949;159:563-4.

2. Agrawal A, Palkar A, Talwar A. The multiple dimensions of Platypnea-Orthodeoxia syndrome: A review. Respir Med 2017;129:31-8.

3. Altman M, Robin ED. Platypnea (diffuse zone I phenomenon?) N Engl J Med 1969;281:1347-8.

4. Strunk BL, Cheitlin MD, Stulbarg MS, et al. Right-to-left interatrial shunting through a patent foramen ovale despite normal intracardiac pressures. Am J Cardiol 1987;60:413-5.

5. Thomas JD, Tabakin BS, Ittleman FP. Atrial septal defect with right to left shunt despite normal pulmonary artery pressure.
J Am Coll Cardiol 1987;9:221-4.

6. Schnabel TG, Ratto O, Kirby CK, et al. Postural cyanosis and angina pectoris following pneumonectomy: relief by closure of an interatrial septal defect. J Thorac Surg 1956;32:246-50.

7. Mercho N, Stoller JK, White RD, et al. Right-to-left interatrial shunt causing platypnea after pneumonectomy. Chest 1994;105:931-3.

8. Schneider B, Hofmann T, Justen MH, et al. Chiari's network: normal anatomic variant or risk factor for arterial embolic events? J Am Coll Cardiol 1995;26:203-10.

9. Rodríguez-Roisin R, Krowka MJ. Hepatopulmonary syndrome-a liver-induced lung vascular disorder. N Engl J Med 2008;358:2378-87.

10. Thomas JD, Tabakin BS, Ittleman FP. Atrial septal defect with right to left shunt despite normal pulmonary artery pressure. J Am Coll Cardiol 1987;9:221-4.

11. Dear WE, Chen P, Barasch E, et al. Sixty-eight-year-old woman with intermittent hypoxemia. Circulation 1995;91:2284-9.

12. Savage EB, Benckart DH, Donahue BC, et al. Intermittent hypoxia due to right atrial compression by an ascending aortic aneurysm. Ann Thorac Surg 1996;62:582-3.

13. Popp G, Melek H, Garnett AR. Platypnea-orthodeoxia related to aortic elongation. Chest 1997;112:1682-4.

14. Laybourn KA, Martin ET, Cooper RA, et al. Platypnea and orthodeoxia: shunting associated with an aortic aneurysm. J Thorac Cardiovasc Surg 1997;113:955-6.

15. Eicher JC, Bonniaud P, Baudouin N, et al. Hypoxaemia associated with an enlarged aortic root: a new syndrome? Heart 2005;91:1030-5. 